\title{
Ion-Cyclotron-Resonance Mass Spectrometry With a Microwave Plasma Source
}

\author{
James B. Friedmann, J. Leon Shohet, Fellow, IEEE, and Amy E. Wendt, Member, IEEE
}

\begin{abstract}
The goal of this work is to couple an electron-cyclotron resonance (ECR) plasma-processing reactor directly to an omegatron mass spectrometer. In the conventional omegatron, ions are formed as the result of ionization of a gas by a narrow electron beam passing through the center of the analyzer along the magnetic field. In this work, an ECR plasma is created in a chamber that is coupled to the omegatron through a small grounded orifice. Ions created in the ECR chamber flow along the magnetic field into the omegatron analysis cell, and the mass spectrum of these ions is recovered. The mass resolution as a function of the omegatron excite voltage for $\mathrm{He}$ and $\mathrm{N}_{2}$ plasmas is obtained and compares well to theoretical calculations.
\end{abstract}

\section{INTRODUCTION}

$P^{\prime}$ ASMA processing of materials is used in a wide variety of industrial applications, and, in many cases, plasma processing is the only method for fabrication of certain products. This is especially the case for integrated-circuit processing, where small linewidths require plasma processing in many of the manufacturing steps. It is often necessary to take in situ spatial, temporal, quantitative, and qualitative measurements of one or more species during processing in order to ensure uniformity, facilitate endpoint detection, and thereby enhance the quality and yield of manufactured products. This information is essential to the understanding of plasma-surface interactions, plasma chemistry, and the development of new plasma-processing applications. The purpose of this work is to investigate the use of an omegatron mass spectrometer [1]-[6] as a diagnostic device for determining electron-cyclotron resonance (ECR) plasma properties and for process monitoring.

Use of ECR plasmas may offer a number of advantages over other plasmas used in processing for some applications. Features of ECR plasmas are: (i) Lower operating pressures resulting in less scattering of ions; (ii) totally electrodeless plasma generation and greater uniformity; and (iii) higher plasma densities with a concomitantly higher free-radical density for a fixed operating process.

Previous work [7] demonstrated the feasibility of coupling a Fourier Transform Mass Spectrometer (FTMS) system directly to an ECR plasma reactor. In this work, we now couple an ECR plasma-processing reactor directly to an omegatron mass spectrometer. The omegatron, which is simpler to use and less costly than a FTMS system, requires a dc magnetic field for operation and hence is well suited for the study of ECR plasmas, since they also require a dc magnetic field. The ECR plasma is not

Manuscript received June 13, 1990; revised October 23, 1990. This work was supported in part by the IBM Corporation, and in part by the National Science Foundation through Grant ECD-8721545.

The authors are with the Engineering Research Center for Plasma-Aided Manufacturing, University of Wisconsin-Madison, 1415 Johnson Drive, Madison, WI 53706.

IEEE Log Number 9041512. perturbed by the spectrometer, since the electric fields of the omegatron are shielded from the plasma by a grounded plate. The only coupling between the plasma and the omegatron is through a small orifice. Other mass spectrometers, such as quadrupoles, often must be shielded from the ECR magnetic field and cannot be placed directly in contact with the plasma. The concept of the omegatron is straightforward: Ions are excited at their various cyclotron resonances, and the current induced when their orbital radii increase and strike a collector is measured. With this technique we have been able to recover mass spectra of both single (He) and two component $\left(\mathrm{N}_{2}\right.$ and $\mathrm{N})$ ECR plasmas created in a chamber that is coupled to the omegatron through a small orifice. We have examined the massresolution capabilities of the omegatron, both theoretically and experimentally.

\section{Operational Theory of the OMegatron}

The omegatron is a device invented by Sommer et al. [1] to measure the charge-to-mass ratio of ions in a high-vacuum system through the use of cyclotron resonance. In an omegatron, an RF electric field is set up normal to a uniform dc magnetic field. A simplified schematic diagram of an omegatron can be seen in Fig. 1. A narrow cylindrical ion beam, flowing parallel to the magnetic field, enters the omegatron analysis cell onaxis. When the frequency of the RF electric field is set to be resonant with the cyclotron motion of the ions, the ions will receive energy from the electric field and will therefore travel in orbits of constantly increasing radius until they strike the walls of the cell. Nonresonant ions may initially gain energy, but will then experience a loss of energy as their velocities become out of phase with the applied RF electric field. Thus the orbit radii of these nonresonant ions will increase for a number of revolutions, then decrease, then increase again, etc., forming contracting and expanding spirals of limited radial extent. Thus an ion collector, placed at an appropriate radius in the analysis cell, should collect only the resonant ions. If the frequency of the applied RF electric field is swept, a mass spectrum of the ion beam can be recovered by plotting the ion current versus the electric field frequency. It is standard practice in omegatron measurements to apply small trapping voltages $(\approx 10 \mathrm{~V})$ to the plates of the analysis cell which are perpendicular to the incoming ion beam and magnetic field. These trapping voltages increase the residence times of the ions in the cell, thereby allowing the applied RF field to act on the ions over a greater number of cycles. This increases the signal-to-noise ratio of the system.

\section{DESCRIPTION OF THE EXPERIMENT}

The omegatron analysis cell is cubical and constructed of $0.127-\mathrm{cm}$ thick, $33 \%$ perforated, 304 stainless-steel sheet. Each 


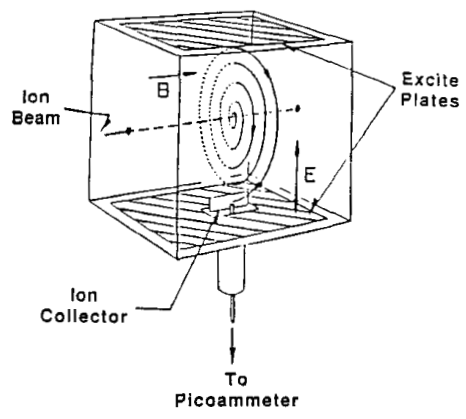

Fig. 1. Schematic diagram of an omegatron.

face of the cell is $13.33 \mathrm{~cm}$ on a side. The plates are electrically isolated from each other through the use of Macor supports. Each face of the cell is connected to a BNC vacuum feedthrough with 20-gauge Kapton-coated copper wire. The ion collector is a rectangular stainless-steel plate with dimensions of 2.5 by 10.0 by $0.20 \mathrm{~cm}$. It is oriented axially in the cell at a radius of $4.5 \mathrm{~cm}$ (see Fig. 2).

During operation of the system, the trapping voltages on the front plate (the cell plate oriented perpendicular to the axial magnetic field and nearest to the incoming ion beam) and the back plate (the cell plate that is opposite the front trap plate) are adjusted to give a maximum peak height at fixed RF electric fields and operating pressures. Typically, the front-plate voltage is set to between 3.5 and $7 \mathrm{~V}$, and the back plate is set to $14 \mathrm{~V}$. The current supplied to the magnet coils is monitored with a $50 \mathrm{mV} / 1000 \mathrm{~A}$ current shunt. The magnet current may range between 980 and $1020 \mathrm{~A}$, giving an axial magnetic field strength of between 0.051 and $0.053 \mathrm{~T}$ in the center of the analysis cell.

The arrangement of the electronics for the omegatron system can be seen in Fig. 3. The setup utilizes two function generators, a picoammeter, two variable dc supplies for the trapping voltages, and an $x-y$ plotter. One function generator is set to generate a triangular wave, and the second function generator creates the RF excite signal that is applied to two parallel plates of the cell, as shown in Fig. 1. Calculations of the RF electric field between the axis and ion collector indicate a variation of $\approx 4 \%$. The excite frequency was varied from 2 to $250 \mathrm{kHz}$ in the He discharge and from 2.0 to $70 \mathrm{kHz}$ in the $\mathrm{N}_{2}$ discharge in order to sweep over the appropriate range of ion-cyclotron-resonance frequencies. The wave-form amplitude of the RF excite generator may range between 2.40 and $37.0 \mathrm{~V}$, corresponding to RF electric-field amplitudes of from 0.180 to $2.78 \mathrm{~V} / \mathrm{cm}$. Its frequency is controlled by the triangular wave. A voltage proportional to frequency is sent to the $x$-axis of the plotter. The ion collector-plate current is monitored with the picoammeter, and this signal is connected to the $y$-axis of the plotter. As the excite frequency is varied, a plot of ion collector current versus the excite frequency is recovered.

A diagram of the complete experiment is shown in Fig. 4. The system was designed to meet the following two criteria: (i) A high-pressure differential was needed between the plasma and analysis regions of the vacuum chamber; and (ii) the same magnetic field was needed for both omegatron analysis and ECR plasma production.

A ring baffle, welded in the chamber at the position indicated in Fig. 4, creates two separate regions in the vacuum chamber. A flange, with a small pinhole orifice $(0.040 \mathrm{~cm}$ in diameter

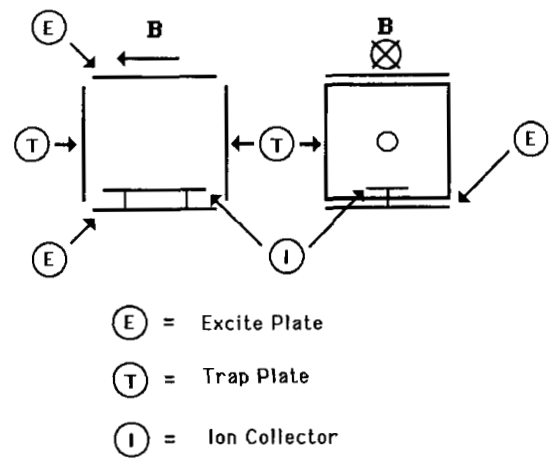

Fig. 2. Orientation of the ion-collector plate in the analysis cell

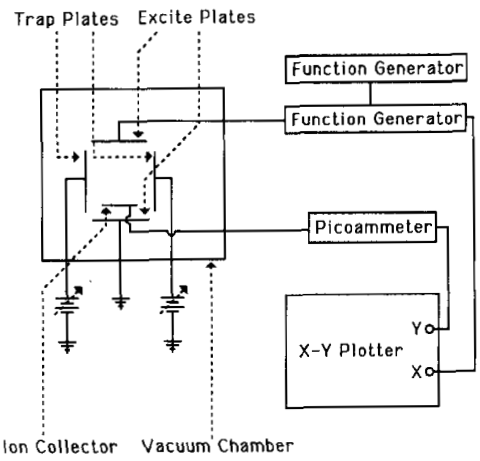

Fig. 3. Configuration of the electronics during omegatron operation.

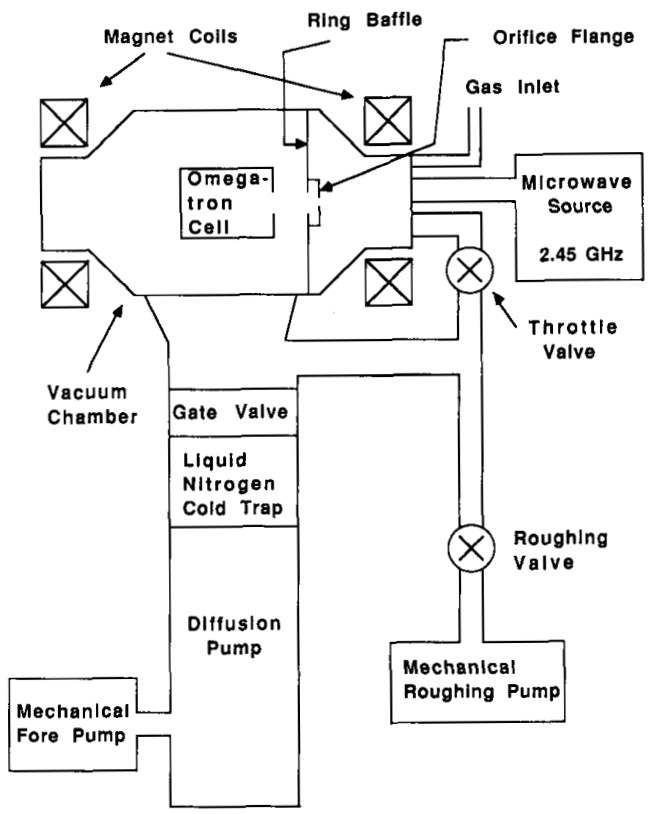

Fig. 4. Diagram of the experiment.

and $0.16 \mathrm{~cm}$ in depth) is bolted to the baffle and covers the hole in the ring. The orifice size can be changed by replacing the flange, thereby changing the conductance and hence the obtainable pressure ratio between the two regions of the vacuum 
chamber. When using the omegatron over a large mass range it may be necessary to increase the ratio of the radius of the pinhole orifice to its depth in order to reduce the mass selectivity of the orifice. To facilitate the pump-down of the entire system, the plasma production regions is connected to the omegatron analysis chamber with a $3.81-\mathrm{cm}$ diameter tube, which can be closed off with a butterfly valve during experiments. The base pressure was $5 \times 10^{-7}$ torr. During operation of the system, plasma is produced at pressures in the neighborhood of $1 \times$ $10^{-2}$ torr, and the analysis region is maintained at pressure of between 2 and $8.5 \times 10^{-5}$ torr.

The magnetic field for both ECR plasma production and ioncyclotron resonance in the analysis cell is provided by a set of coils arranged in a magnetic-mirror configuration. The coils are constructed of hollow copper tubing with a rectangular cross section of dimensions 2.54 by $1.91 \mathrm{~cm}$. Each coil consists of 4 rows of 12 turns, for a total of 48 turns. The coils have an outer diameter of $68.0 \mathrm{~cm}$, and their centers are separated axially by a distance of $61.5 \mathrm{~cm}$.

A numerical calculation of the magnetic field has been made. A multiturn model was used to simulate the two sets of 48-turn coils. A plot of the results of the simulation can be seen in Fig. 5 for a current of $1000 \mathrm{~A}$. From the calculation it can be seen that there is a magnetic field variation of $7 \%$ along the axis of the omegatron cell. Measurements of the field with a gaussmeter gave values of $0.054 \mathrm{~T}$ in the center and $0.130 \mathrm{~T}$ in the coil throat for a magnet current of $1020 \mathrm{~A}$. These values agree to within $0.5 \%$ of the results of the numerical calculation. Calculations of the radial magnetic field variation, at the mid-plane of the chamber, indicate a field variation of $\approx 3 \%$.

Plasma is generated at electron-cyclotron resonance at a frequency of $2.45 \mathrm{GHz}$. Microwaves are produced by a magnetron source and fed into the vacuum chamber through a waveguide quartz window. The microwave source produces a full-wave rectified signal at $60 \mathrm{~Hz}$ and has an average power output of 50 to $2500 \mathrm{~W}$. Typically, plasma can be produced with coupled powers of approximately $70 \mathrm{~W}$. Since the wavelength of the microwaves is $\approx 12 \mathrm{~cm}$, they are effectively shielded from the omegatron analysis region by the grounded ring baffle which contains the orifice.

\section{RESUlTS AND Discussion}

\section{A. Ion Current Measurements}

For a nitrogen plasma, measurements were made of the ion current flowing from the plasma source into the analysis region. By biasing the front or back plate into ion saturation while grounding all the other plates, the ion current to that plate could be measured. With the pressure in the analysis region at $7.4 \times$ $10^{-5}$ torr and the pressure in the plasma region at $1 \times 10^{-2}$ torr, the back plate was biased to $-50 \mathrm{~V}$ and all the other plates were grounded. An ion current of $3 \times 10^{-8} \mathrm{~A}$ (assuming a singly ionized charge state, this corresponds to $3 \times 10^{11}$ ions $/ \mathrm{s}$ ) was measured. Biasing the front plate to $-50 \mathrm{~V}$ and grounding the others gave an ion current of $7.2 \times 10^{-8} \mathrm{~A}\left(7.2 \times 10^{11}\right.$ ions $/ \mathrm{s}$ ).

The signal-to-noise ratio of the signals measured by the picoammeter depended upon the trapping and excite voltages. This is because the ions experience, at most, a single pass back and forth between the trapping plates. Consequently, there is a competing effect between the time it takes the ions to traverse the cell in the axial direction and the time it takes for the excite signal to increase the radii of the ions so that they hit the col-

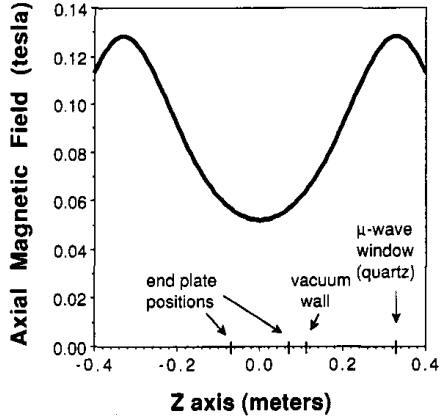

Fig. 5. Calculation of the magnetic field for a current of $1000 \mathrm{~A}$.

lector. During optimum running conditions (largest signal) the signal-to-noise ratio was on the order of 100 . When the excite signal voltage was too low, relative to the trapping voltages, the ions exited the cell before they could strike the collector, and consequently no signal was seen.

\section{B. Mass Spectra of $\mathrm{N}_{2}^{+}$and $\mathrm{He}^{+}$Ions}

The mass spectra of two different gases (nitrogen and helium) were measured with the omegatron, and representative spectra can be seen in Figs. 6 and 7. The data for these spectra were taken from plots of the ion-collector current versus the excitation frequency. To obtain a mass spectrum, the excitation frequency was converted to a mass value by using the definition of the ion-cyclotron-resonance frequency (see equation (2)) and assuming a singly ionized charge state for the ions. The dc signals displayed in Figs. 6 and 7 have been filtered by the picoammeter, eliminating a large ac component (compared to the $\mathrm{dc}$ current generated by the resonant ions striking the collector) at the excitation frequency. This ac component, which is also observed in the absence of plasma, is due to capacitive coupling of the excitation signal to the ion collector. Since the capacitively coupled signal can be as much as $10^{4}$ times greater than the dc signal of the ions, it is very important that the picoammeter filter out any signal at the excite frequency.

The nitrogen plasma spectrum (Fig. 6) exhibits three different peaks, centered at 28,17 , and 13 amu. For this case, the value of the magnetic field in the center of the analysis cell was 0.051 $\mathrm{T}$. The line at 28 amu corresponds to singly-ionized $\mathrm{N}_{2}^{+}$. The full-width at the base of this line is $12 \mathrm{amu}$, giving a mass resolution $(\mathrm{m} / \Delta \mathrm{m})$ of 2.3. The small peak at $13 \mathrm{amu}$ corresponds to a combination of $\mathrm{N}_{2}^{++}$and $\mathrm{N}^{+}$, both of which have a chargeto-mass ratio of 14 . The $17 \mathrm{amu}$ line is most likely due to water vapor present in the system.

The helium plasma spectrum (Fig. 7), taken at a RF electric field amplitude of $1.65 \mathrm{~V} / \mathrm{cm}$ and a magnetic field strength of $0.052 \mathrm{~T}$, exhibits a large peak at $3.9 \mathrm{amu}$, which corresponds to singly ionized $\mathrm{He}^{+}$. The percent difference between the measured and predicted mass of the peak is $2.5 \%$. The full-width of the base of the helium line is $0.71 \mathrm{amu}$, yielding a mass resolution $(m / \Delta m)$ of 5.5

\section{Experimental Measurements of the Mass Resolution}

The theoretical mass resolution of the omegatron is given by [1]

$$
m / \Delta m=q B^{2} r_{0} / 2 m E_{0}
$$




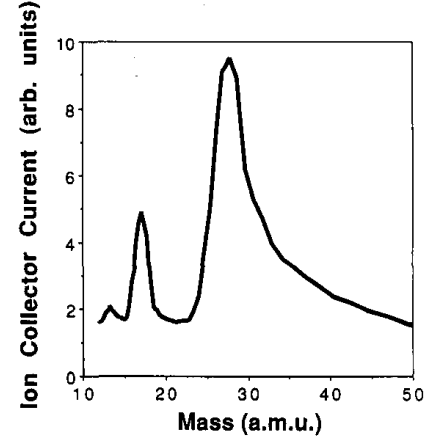

Fig. 6. Mass spectrum of a $\mathrm{N}_{2}$ plasma.

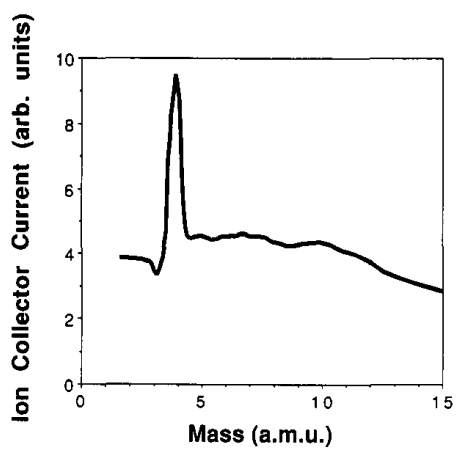

Fig. 7. Mass spectrum of a He plasma.

where $E_{0}$ is the amplitude of the RF electric field applied to the excite plates, $\Delta m$ is the width of the ion peak in mass units, $B$ is the magnetic field strength, $q$ is the ion charge, and $r_{0}$ is the ion-collector radius $(0.045 \mathrm{~m})$. Thus the higher $m / \Delta m$, the better the mass resolution of the system.

From the above expression it can be seen that the omegatron mass resolution should increase for decreasing RF electric fields. In addition, the magnetic field nonuniformity places an upper limit on the achievable mass resolution of the system, as will be described later.

In order to examine the mass resolving capabilities of the system, measurements of the mass resolution $(m / \Delta m)$ as a function of the excitation electric field were made for a nitrogen and a helium plasma (see Figs. 8 and 9). The maximum mass resolution for a nitrogen plasma was measured to be 2.3 at an electric field of $0.975 \mathrm{~V} / \mathrm{cm}$. The maximum experimentally measured mass resolution for a helium plasma was 8.2 at an electric field of $0.375 \mathrm{~V} / \mathrm{cm}$.

\section{Comparison with Theory}

The cyclotron frequency of an ion is inversely proportional to its mass $m$ :

$$
\omega_{c}=2 \pi f_{c}=q B / m
$$

so higher mass species have lower cyclotron resonant frequencies. Thus the mass resolution, $m / \Delta m=\omega_{c} / \Delta \omega_{c}$, sets an upper limit on the observable mass. This upper limit is extremely important, because it determines which chemical species the omegatron mass spectrometer can detect.

The mass resolution of the omegatron was given in (1). The radius of the ion collector is $r_{0}=0.045 \mathrm{~m}$. In the operating

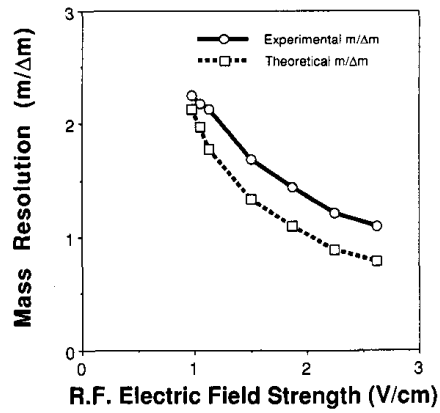

Fig. 8. Mass resolution as a function of RF electric field amplitude for $\mathrm{N}_{2}^{+}$ions.

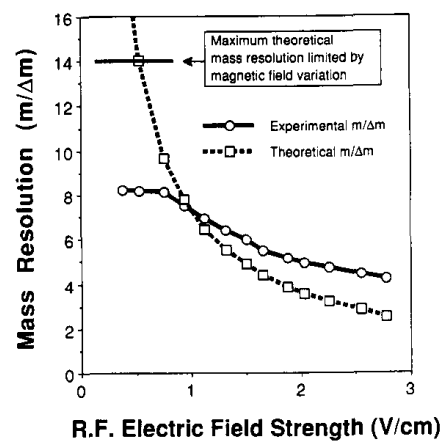

Fig. 9. Mass resolution as a function of RF electric field amplitude for $\mathrm{He}^{+}$ions

field of $0.052 \mathrm{~T}$ with an electric field amplitude of $E_{0}=0.975$ $\mathrm{V} / \mathrm{cm}$, equation (1) predicts a mass resolution for $\mathrm{N}_{2}^{+}$of $m / \Delta m$ $\approx 2.1$, giving a $9.5 \%$ difference between theory and experiment. Measurements of mass resolution at higher electric field strengths (up to $2.80 \mathrm{~V} / \mathrm{cm}$ ) show the predicted scaling from (1).

In principle, the mass resolution can be improved by lowering the amplitude of the excitation field until an upper limit determined by the magnetic field variation is reached. This limiting value of $m / \Delta m$ is $\approx 14$, since there is a magnetic field variation of $\approx 7 \%$ across the analysis cell. From (1) it can be seen that this limit should be reached for nitrogen when the amplitude of the excitation electric field is $0.293 \mathrm{~V} / \mathrm{cm}$.

The mass resolution for $\mathrm{N}_{2}^{+}$ions was measured for excitation electric fields from 2.78 down to $0.975 \mathrm{~V} / \mathrm{cm}$. And thus no limiting value of resolution was observed, both as shown in Fig. 8 and as predicted by (1). However, for the case of $\mathrm{He}^{+}$ the mass resolution was limited to a value of $\approx 8.2$ for excitation electric fields of less than $0.750 \mathrm{~V} / \mathrm{cm}$, suggesting that the mass resolution limit has been reached. This compares to a value of $0.510 \mathrm{~V} / \mathrm{cm}$ predicted by (1) and the magnetic field variation of $7 \%$. The difference between the theoretical and experimental mass limits could be due to the radial variation of the magnetic field and the RF electric field variation, which we did not consider when estimating the theoretical mass limit.

In order to increase the mass resolution of the omegatron, a stronger and more uniform magnetic field is needed in the analysis region. By choosing a maximum allowable linewidth of 1 amu (i.e., maintaining the capability to resolve ions with a mass difference of $1 \mathrm{amu}$ ), it is possible to calculate the magnetic 
field strength needed for various masses. Given an ideal situation, where the magnetic field is completely uniform and the excitation voltage is $1 \mathrm{~V}$, the mass resolution is $m / \Delta m \approx 20$ for an argon plasma with a $0.052-\mathrm{T}$ field strength in the cell. For $\mathrm{CF}_{4}$ ions under the same conditions, the mass resolution is $m / \Delta m \approx 9$. By doubling the magnetic field to $0.104 \mathrm{~T}$, the mass resolution for $\mathrm{CF}_{4}$ ions can be increased to $m / \Delta m \approx 36$.

\section{Conclusion}

We have demonstrated that it is feasible to couple an ECR plasma source directly to an omegatron spectrometer. Since the same dc magnetic field is used for both plasma production and mass analysis, the omegatron diagnostic does not perturb the plasma. Mass spectra of various plasmas have been measured and the experimental results correspond well to theoretical predictions.

Improved mass resolution can be obtained with the omegatron mass spectrometer with a stronger and more uniform magnetic field in the analysis region. This will allow the omegatron to be used with ECR plasmas covering a wider mass range.

Some applications for the omegatron in a manufacturing environment would be for detecting the completion of an etching process (end-point detection). That is, detecting the presence of ions from the base material as the etching process "breaks through" the material being etched. In addition, it can be used to monitor ion-species concentrations during a reaction.

\section{ACKNOWLEDGMENT}

The advice and assistance of D. T. Anderson and F. S. B. Anderson are greatly appreciated.

\section{REFERENCES}

[1] H. Sommer, H. A. Hipple, and J. A. Thomas, "The measurement of e/m by cyclotron resonance," Phys. Rev., vol. 82, pp. 697702 , June 1951.

[2] C. E. Berry, "Ion trajectories in the omegatron," J. Appl. Phys., vol. 25, pp. 28-31, Jan. 1954.

[3] D. Lichtman, "Use of the omegatron in the determination of parameters affecting limiting pressures in vacuum devices," $J$. Appl. Phys., vol. 31, pp. 1213-1221, July 1960.

[4] B. W. Petley and K. Morris, "An omegatron with linear orbit drift giving improved resolution without loss of sensitivity," J. Sci. Instrum., vol. 42, pp. 492-494, Apr. 1965.

[5] 1. Marklund and H. Danielsson, "Influence of collection efficiency on omegatron measurements," Rev. Sci. Instrum., vol. 37, pp. 319-325, Mar. 1966.

[6] E. Y. Wang, L. Schmitz, Y. Ra, B. LaBombard, and R. W. Conn, "An omegatron mass spectrometer for plasma ion species analysis," Rev. Sci. Instrum., vol. 61, pp. 2155-2158, Aug. 1990.

[7] J. L. Shohet et al., "A plasma source for Fourier transform mass spectrometry," Plasma Chem. Plasma Process., vol. 9, pp. 207 210 , Oct. 1989.

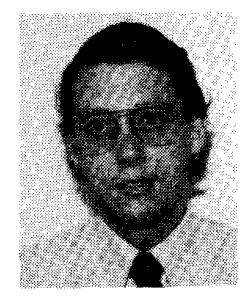

James B. Friedmann was born in South Saint Paul, MN, on October 19,1964 . He received the B.A. degree (physics) from Carleton College in 1987, and the M.S. degree (electrical engineering) from University of WisconsinMadison in 1990, where he is currently pursuing the Ph.D. degree. His research interests include ECR plasmas, plasma processing, and plasma modeling.

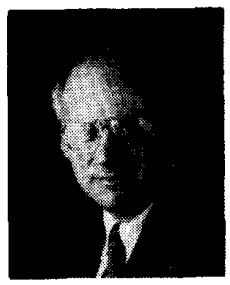

J. Leon Shohet (S'56-M'62-SM'72-F'78) received the Ph.D. degree electrical engineering from Carnegie-Mellon University in 1961.

He served on the faculty of The Johns Hopkins University before joining the University of Wisconsin faculty in 1966, and was appointed Professor of Electrical and Computer Engineering in 1971 . He currently serves as the Director of the University's NSF Engineering Research Center for Plasma-Aided Manufacturing and the Torsatron/Stellarator Laboratory. $\mathrm{He}$ is the author of a textbook on plasma science and over 90 journal articles and more than 300 conference papers. He holds five patents. His research interests are in plasma-aided manufacturing; fusion, especially waves, instabilities, heating, confinement, and diagnostics; communications; magnetohydrodynamics; electromagnetic field theory; biophysics; quantum electronics; and lasers.

Dr. Shohet is a Fellow of the American Physical Society. He received the Frederick Emmons Terman award of the American Society of Engineering Education, the Merit Award of the IEEE's Nuclear and Plasma Sciences Society, the IEEE Centennial Medal, and the IEEE Plasma Science Prize.

Amy E. Wendt (M'89) was born in Richmond, CA, in 1960. She received the B.S. degree (engineering and applied science) from the California Institute of Technology in 1982, and the M.S. and Ph.D. degrees (electrical engineering and computer science) from the University of California at Berkeley in 1985 and 1988, respectively.

She was a Research Associate at the Engineering Research Center for Plasma-Aided Manufacturing at the University of Wisconsin-Madison from 1988 to 1990. She is currently an Assistant Professor in electrical and computer engineering at the University of WisconsinMadison. Her research interests include the use of plasmas for materials processing. 